International Research Journal of Materials Sciences and Applications

(ISSN:2575-1034)

\title{
Role of Dispersion and Functionalization on Mechanical Properties in Carbon Nanotube-Polymer Composites
}

\author{
Sai Praveen Kumar Medisetti ${ }^{1}$ and Dr. Nicholas Roberts ${ }^{1}$
}

${ }^{1}$ Department of Mechanical and Aerospace Engineering, Utah State University, Logan, Utah 84322, USA

\section{ABSTRACT}

Carbon nanotubes (CNTs) exhibit excellent mechanical and thermal properties. Designing composites that employ CNTs as the reinforcing or filler material offer the potential to create bulk materials with greatly enhanced mechanical and thermal properties. Unfortunately, the resulting property enhancement in CNT, and other carbon nanomaterial, enhanced composites vary greatly. In macroscale composites, like carbon fiber/epoxy composites, the large interface area and relatively low surface area to volume ratio of the carbon fiber/epoxy results in excellent transfer of load or thermal energy across the interface, thus allowing the carbon fiber to enhance the mechanical or thermal properties of the composite. In nanocomposites, the high surface area to volume ratio between the reinforcing and matrix materials requires tightly coupled interactions at the interface. Additionally, due to the high surface area to volume ratio of the nanomaterial filler, there is added difficulty in ensuring the reinforcing materials are uniformly dispersed. These two major differences between macroscale and nanoscale composites results in the existing predictive models failing to predict the effective composite properties. To improve the understanding of the roles that interface bonding and the dispersion of the reinforcing material play on the effective properties, we present the results of a detailed experimental study. To study the role of the reinforcing material/matrix interface bonding, we fabricate CNT/polymer composites where the CNTs are functionalized with different functional groups. To study the role of the nanoparticle filler dispersion, we fabricate CNT polymer composites with different dispersing techniques. This work shows that CNT dispersion is critical for fabricating CNT composites with enhanced mechanical properties.
*Correspondence to Author:

Dr. Nick Roberts

Assistant Professor, Mechanical and Aerospace Engineering, Utah State University

Email: nick.roberts@usu.edu

How to cite this article:

Sai Praveen Kumar Medisetti and Nicholas Roberts. Role of Dispersion and Functionalization on Mechanical Properties in Carbon Nanotube-Polymer Composites. International Research Journal of Materials Sciences and Applications, 2017; 1:3.

\section{eSciencePublisher}

eSciPub LLC, Houston, TX USA. Website: http://escipub.com/ 


\section{INTRODUCTION}

Polymers have a wide range of applications in the commercial industry due to properties like flexibility, cost, and processability. Two emerging nanoscale applications areas for polymers are in thermal interface materials (TIM) and nanofabrication in soft lithography (1). TIMs are currently employed for improving the thermal conductance across an imperfect interface (CPU package and a heat sink), where high thermal conductivity and highly flexible materials are preferred. In nanoscale soft lithography, there is a push to create the smallest size features possible. For both of the applications even though the mechanical properties are not of primary importance, wider usage is limited by the low modulus of the polymers (2). Despite flexibility being a critical criterion for the usage of polymers, higher flexibility can be a detrimental limit in certain applications. There must be a balance between the flexibility and other desired properties like thermal, mechanical, and processability. For TIMs, flexibility is one of the important requirements but low modulus leads to low stress transfer rates and raises the issue of long term reliability. An optimum level of strength is also required for ideal TIMs. In recent years there has been a substantial increase in the cooling requirement for CPUs in the micro-electronics industry. A lot of research has been done to efficiently transfer heat generated by CPUs while often neglecting mechanical properties. Currently there is a need for TIMs, which have good mechanical properties along with the enhanced thermal properties.

In soft lithography, polymer stamps having features to transfer the pattern are nanofabricated using conventional methods like molding with the help of polydimethylsiloxiane (PDMS) (3). Nanofabrication enables the generation of reproducible patterns with sizes down to several nanometers (2). This commercial application of nanofabrication is widely used in the semiconductor industry and recently there has been demand for below $10 \mathrm{~nm}$ sizes in pattern making (3) (2). The application of a polymer for below $100 \mathrm{~nm}$ sizes is limited partially the low elastic modulus of polymers as the low strength inhibits release of the element from the stamps (2), thereby raising the need for better mechanical characteristics of nanoscale fabrication without deformation or failure (2).
For the purpose of balance between flexibility and strength, a composite is needed where in the flexibility of the polymer can be utilized with the strength enhanced through the addition of a filler material. Since the discovery of carbon nanotubes (CNT) by lijima in 1991 (4), a vast amount of research has been done to utilize their superior mechanical and thermal properties in materials and devices. Along with their superior mechanical properties, CNT are known for their ease of fabrication [need citation]. With the processability and flexibility of polymers and the mechanical strength and high thermal conductivity of CNT, CNT/polymer composite are an ideal composite for applications mentioned above. In order to achieve the maximum enhancements of CNT/polymer composites, special care must be taken to ensure adequate dispersion of the CNTs in the matrix and sufficient bond strength between the CNT and the matrix material (5) (6). The objective of this research is to investigate the role of dispersion and functionalization on CNT/polymer composites in enhancing the mechanical properties.

\section{METHOD}

Effective dispersion of CNTs requires overcoming van der Waals forces between the CNTs. For this, mechanical stirring is required and ultrasonication is one of the preferred methods. Ultrasonication is a process of applying sound waves at ultrasonic frequencies to agitate particles. The effectiveness of ultrasonication increases in liquids having low viscosity. The PDMS elastomer polymer has a very high viscosity of $5100 \mathrm{cP}$, which reduces the effectiveness of sonication. For this purpose, a solvent is required for dispersion carbon nanotubes followed by mixing the with the PDMS elastomer polymer prior to curing. Along with viscosity, the stability of the dispersion and the evaporation rate of the solvent are important as they control the final dispersion of the reinforcing material and the amount of time and effort required for composite fabrication, respectively. Solvents like DMF (Dimethylformamide), ethanol, methanol and IPA are tested. Ethanol was found to be the ideal choice as it has a low viscosity of $1.095 \mathrm{cP}(7)$ and high evaporation rate. The three functionalization considered for this research are unfunctionalized, carboxylic functional group $(\mathrm{COOH})$, and hydroxyl functional group $(\mathrm{OH})$. For this study multi-walled car- 
bon nanotubes with $50-80 \mathrm{~nm}$ in diameter and $10-20 \mu \mathrm{m}(8)$ in length and a purity of $95 \%$ are considered. The matrix material used is PDMS, namely Sylgard $₫ 184$ Silicone Elastomer. In this study three different weight percentage of 0.1 , 0.2 and $0.3 \%$ for unfunctionalized CNTs and 0.3 , $0.6,0.9$ weight percentage for $\mathrm{COOH}$ and $\mathrm{OH}$ functionalization was used to understand the role of dispersion and functionalization on mechanical properties of polymer composites.

\section{Fabrication}

As shown in Figure 1, samples for tensile testing were made as per ASTM standard of D638 - 02a. As recommended by Sylgard the PDMS and cross linker are mixed together at the ratio of 10:1. After numerous trials and improvements, the process of making polymer composite was optimized and it involves four steps, namely (1) dispersion, (2) evaporation, (3) degas, and (4) curing. Two different methods were adapted for dispersed samples and non-dispersed samples. The main difference between these samples was the additional ultrasonication step for the dispersed samples. All the other stages of evaporation, degas, and curing were kept the same to better understand the effects of dispersion.

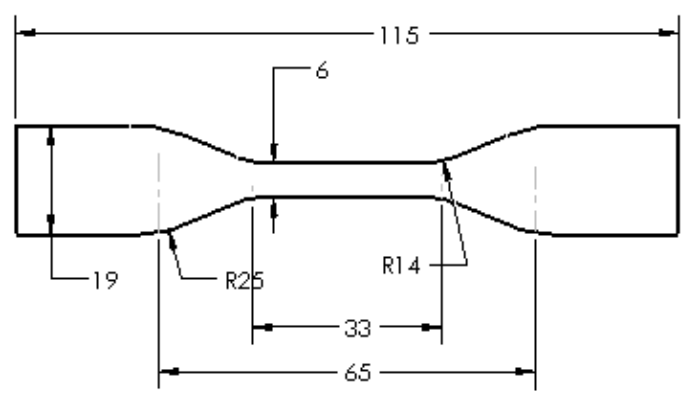

Figure 1: Tensile Testing Sample as per ASTM D638 - 02a Type IV.

\section{a) Dispersion}

A small amount of ethanol used in sonication is more effective as it reduces the evaporation time. To test the samples on the same criteria, the amount of ethanol for all samples was kept constant at $25 \mathrm{ml}$. The measured amount of CNTs calculated using the Eq. 1 is mixed in with ethanol in a $30 \mathrm{ml}$ beaker. The solution is sonicated at $35 \%$ amplitude of $20 \mathrm{kHz}$ frequency for 10 minutes. The pulse on and off time was kept at 10 seconds and 30 seconds, respectively. As shown in Figures 2-4 the suspension of CNTs in the solution was very good even after 5 hours for all the solvents.

$$
C N T=\frac{w t(P D M S+\text { CrossLinker })}{(1-w t)}
$$

Eq. 1

\section{b) Evaporation}

After dispersion CNT solvent solution and polymer solution are mixed together. Thereafter the solution is heated on a hot plate at $120^{\circ} \mathrm{C}$ for 90 minutes. For the first hour the solution is stirred every 20 minutes to let the ethanol evaporate.

\section{c) Degas}

Once the ethanol has evaporated out of the solution, cross linker is mixed at the ratio of 10:1 and hand stirred for 3 minutes. Air bubbles are created during the mixing of PDMS and cross-linker. To remove the air bubbles, the solution is degassed in a vacuum chamber for 40 minutes.

\section{d) Curing}

As recommended by DOW CORNING, the cure time is dependent on temperature (9). For our study we performed the cure process at a temperature of $100^{\circ} \mathrm{C}$. The amount of time required for the mold to reach the desired temperature also depends upon the oven and mold material. As discussed by Johnston (9), the time taken by the mold to reach the desired temperature was determined and added to the curing time of 35 min, recommended by DOW CORNING.

\section{Dispersion Characterization}

Despite technical challenges, there is good amount of research being done on the dispersion of CNTs in various mediums [Ric08]. To quantify the dispersion of CNTs in PDMS, methods were used significantly, namely optical microscopy and electron microscopy. In our study we observed the dispersion using optical microscope and thereafter processed the images using an image processing code [YWu11]. Electron microscopy was attempted due to the small scale of the CNTs, but the images obtained from optical microscopy were more effective in quan- 

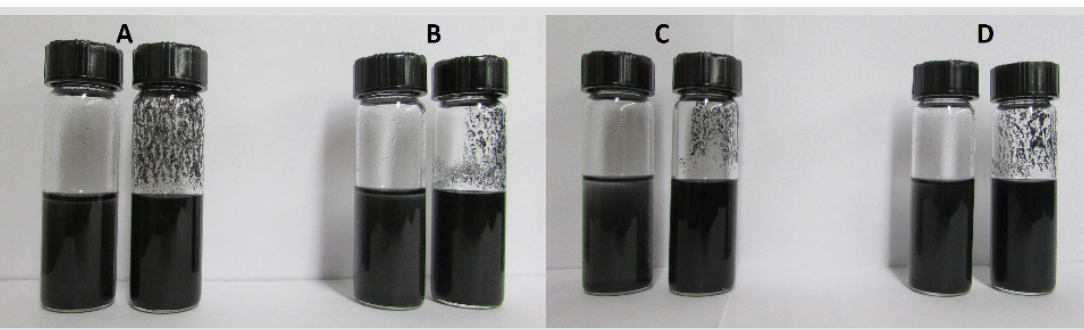

Figure2: Immediately after Sonication (A-DMF, B-Ethanol, C-Methanol, D-IPA)
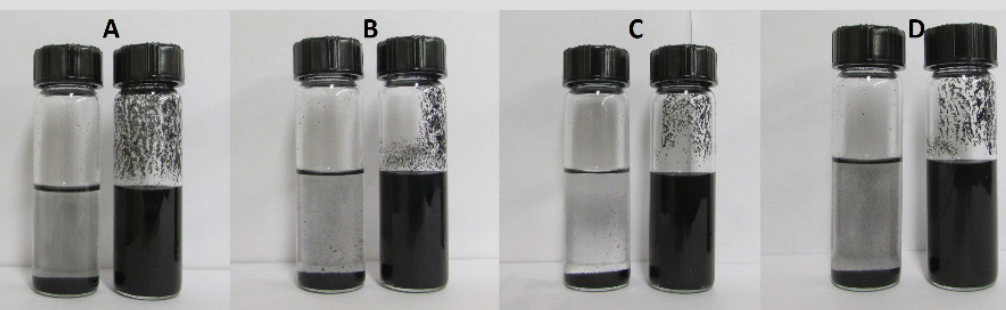

Figure 3: 1 Hour after Sonication (A-DMF, B-Ethanol, C-Methanol, D-IPA)
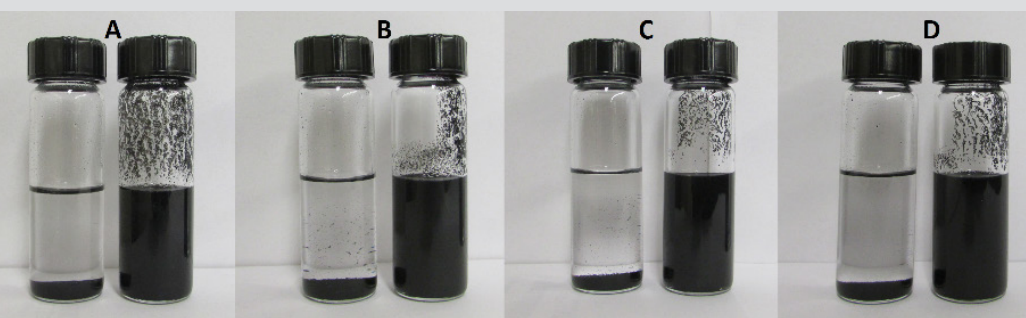

Figure 4: 5 Hours after Sonication (A-DMF, B-Ethanol, C-Methanol, D-IPA)

tifying the CNT dispersion.

\section{RESULTS}

Dispersion of CNTs in the polymer matrix was studied using an optical microscopic. Thereafter the images were converted to greyscale and processed using the image processing code to calculate radii and spacing of the CNTs in polymer matrix. Even though the CNTs cannot be resolved, agglomerated parts of CNTs can be resolved.

To understand the effect of the CNT dispersion and functionalization on mechanical properties, Young's modulus of the CNT/polymer composite at three different percentages of CNTs were determined by tensile testing. Figures 7-10 shows tensile plots for dispersed and non-dispersed CNT-polymer composites of three different functionalization at various weight percentages.

\section{CONCLUSION}

The results of the dispersion characterization clearly show marked improvements in rate of dispersion due to ultrasonication. Figure $6 a$ and Figure $6 \mathrm{c}$ show reduction in clump size from non-dispersed to dispersed samples. Similarly Figure $6 b$ and Figure $3 d$ show huge reduction in spacing between the clumps.

Figure 10 show reduction in Young's modulus due to presence of ethanol in the PDMS matrix, but this reduction as seen it not significant. It was apparent from the tensile results of Instron that the method of ultrasonication enhanced Young's modulus of $\mathrm{COOH}, \mathrm{OH}$ and unfunctionalized CNT polymer composite samples. The enhancement of mechanical property varied with all three functionalization indicating each functionalization behaved differently in bonding with the PDMS polymer. As shown in Figure 7 - Figure $9 \mathrm{OH}$ functionalization gave higher Young's modulus on comparison with $\mathrm{COOH}$ indicating better bonding characteristics. Furthermore, the results indicated unfunctionalized CNT polymer composite exhibited much higher Young's modulus for $0.6 \%$. This increase can be attributed to 

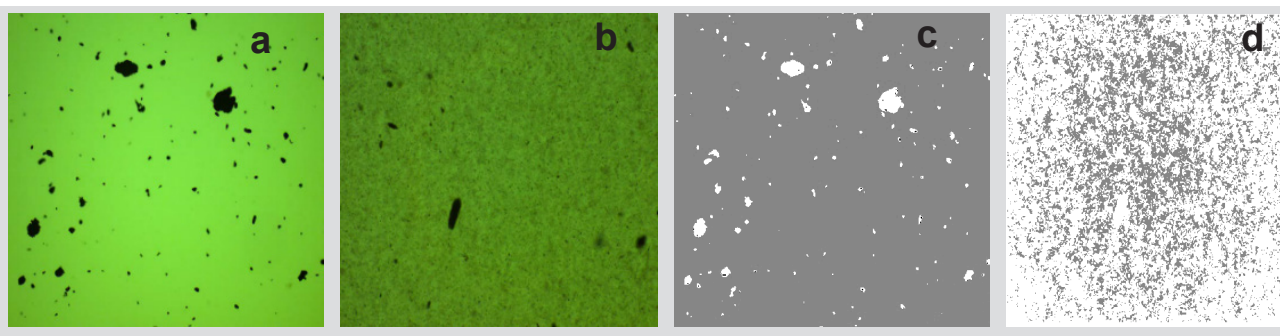

Figure 5: (a) 10X Optical Image of Non-dispersed Sample; (b) 10X Optical Image of Dispersed Sample. c) 10XGreyscale Image of Non-dispersed Sample; (d) 10X Greyscale Image of Dispersed Sample.

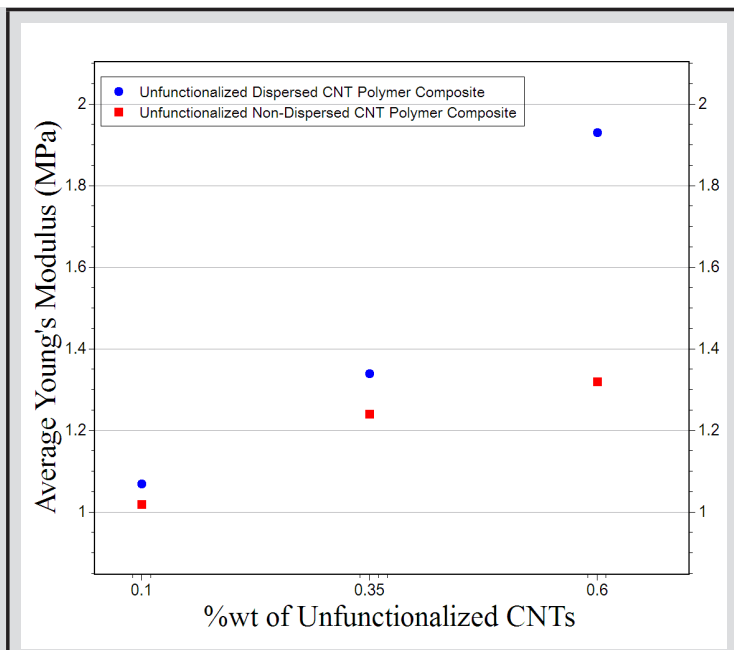

Figure 7: Young's modulus of dispersed and non-dispersed Unfunctionalized Carbon Nanotube Polymer Composite.

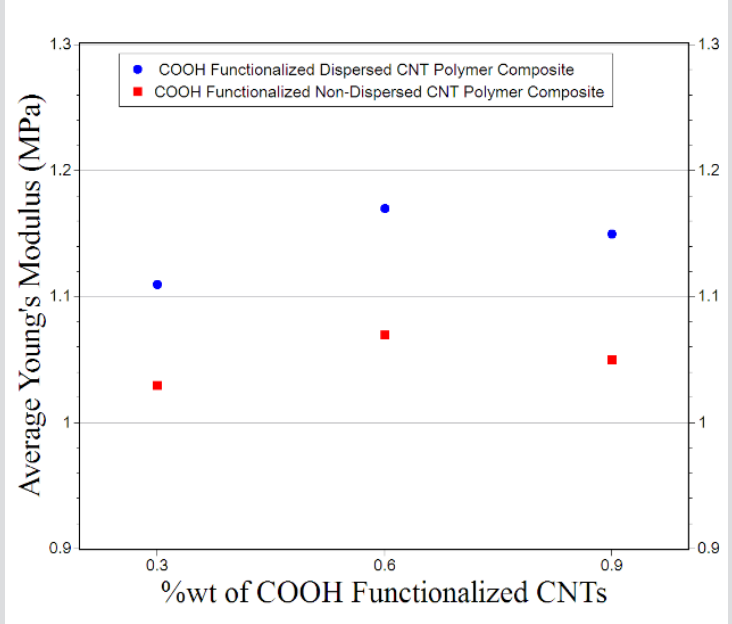

Figure 8: Young's modulus of and non-dispersed $\mathrm{COOH}$ functionalized Carbon Nanotube Polymer Composite.

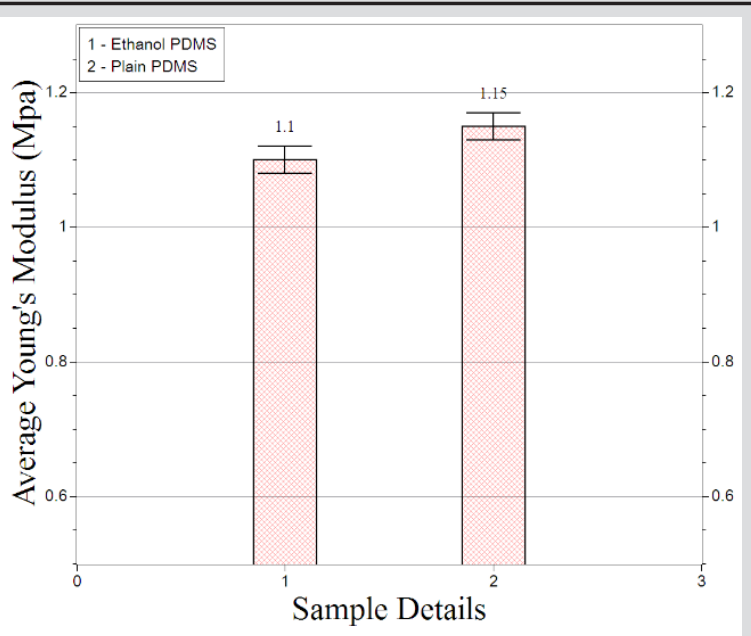

Figure 10: Young's Modulus of Ethanol PDMS and plain PDMS
Figure 9: Young's Modulus of dispersed and non-dispersed $\mathrm{OH}$ functionalized Carbon Nanotube Polymer Composite. 

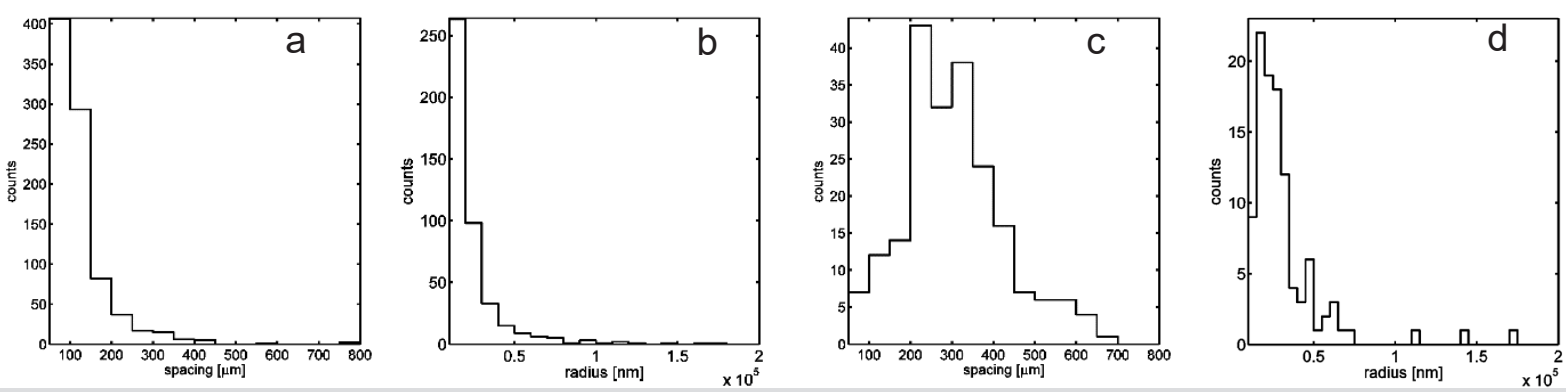

Figure 6: Histogram plot of radii (a) and spacing (b) in non-dispersed $0.1 \%$ unfunctionalized CNT polymer samples. Histogram plot of radii (c) and spacing (d) in dispersed $0.1 \%$ unfunctionalized CNT polymer samples.

difference in density of CNT for three functionalization's therefore unfunctionalized CNTs with lower density had more number of CNTs at the same percentage weight on comparison with $\mathrm{COOH}$ and $\mathrm{OH}$.

\section{References}

1. Tang, S. K. and Whitesides, G. M.,. Basic microfluidic and soft lithographic techniques optofluidics: Fundamentals, Devices and applications. s.I. : McGraw Hill, 2010.

2. Improved pattern transfer in soft lithography using composite stamps. Odom, T. W., Love, J. C., Wolfe, D. B., Paul, K. E., and Whitesides, G. M. 12, 2002, Langmuir, Vol. 18, pp. 5314-5320.

3. Lipomi, D., Martinez, R., Cademartiri, L., and Whitesides, G. Soft Lithographic Approaches to Nanofabrication. s.I. : Elsevier, 2012.

4. Helical microtubules of graphitic carbon. lijima, S. 1991, Nature, Vol. 354, pp. 56-58.

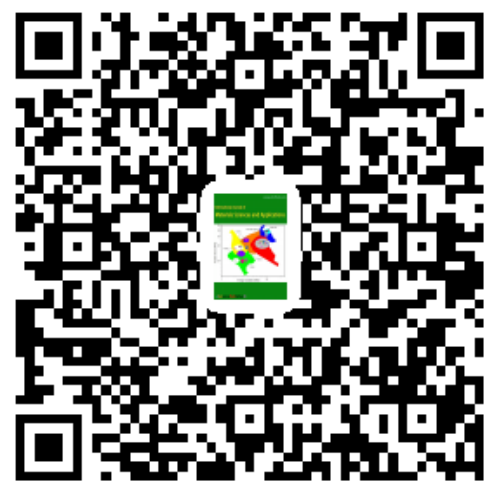

5. Carbon nanotube-polymer composites: chemistry, processing, mechanical and electrical properties. Spitalsky, Z., Tasis, D., Papagelis, K., and Galiotis, C. 3,2010 , Progress in polymer science, Vol. 35 , pp. 357-401.

6. Functionalization of carbon nanotubes. Jeon, I.-Y., Chang, D. W., Baek, J.-B., and Kumar, N. A. 2011, INTECH Open Access Publisher.

7. Solvents, Viscosity of. Accudynetest. Accudynetest. [Online] 10 06, 2016. Accudynetest.

8. Solvents, Viscositysolvents Viscosity of. Nanoamor. [Online] 10 06, 2016. [Cited: 12 15, 2016.] http:// www.nanoamor.com/inc/pdetail?v=1\&pid=1365.

9. Mechanical characterization of bulk Sylgard 184 for microfluidic and microengineering. Johnston, I., McCluskey, D., Tan, C., and Tracey, M. 3, 2014, Journal of Micromechanics and Microengineering, Vol. 24, p. 035017.

10. Y. Fainman, L. Lee, Demetri Psaltis, Changhuei Yang. Optofluidics: Fundamentals, Devices and Applications. s.I. : McGraw-Hill Biophotonics, 2009. 